\title{
ON ENDOMORPHISMS OF ABELIAN TOPOLOGICAL GROUPS
}

\author{
ELI KATZ AND SIDNEY A. MORRIS
}

\begin{abstract}
A family $\Phi$ of continuous endomorphisms of a topological group $G$ is said to be small if for every subgroup $H$ of $G$ of cardinality $\operatorname{card}(H)<$ $\operatorname{card}(G)$ there exists an element $g \in G$ such that $\Phi g \cap H=0$. M. I. Kabenjuk [5] proved that if $G$ is a compact connected Hausdorff abelian group of countable weight then every countable family $\Phi$ of nontrivial endomorphisms of $G$ is small. He asked if "compact" can be replaced by "complete". In this note the answer is given in the negative, but it is shown that "compact" can be replaced by "locally compact".
\end{abstract}

A ring $R$ is said to be representable if it is the endomorphism ring of an abelian group $A$. Clearly to be representable $R$ must be associative and have a 1 . However necessary and sufficient conditions are not known (see Fuchs [1, Chapter XV]). By considering discrete topologies it is immediately clear that the class of rings which are the ring of continuous endomorphisms of an abelian topological group is a wider class of rings. Indeed it has been conjectured that this is the class of all associative rings with 1 . In analyzing this conjecture Kabenjuk [5] runs into the following problem.

Problem. Let $G$ be a complete connected abelian topological group, $H$ a subgroup of $G$ and $\Phi$ a set of nonzero continuous endomorphisms of $G$. Suppose that card $\Phi$, $\operatorname{card}(H)<\operatorname{card}(G)$. Is there an element $g \in G$ such that $\Phi g \cap H=\emptyset$ ?

The example below provides a negative answer to this question. First we need a definition.

DEFINITION. Let $X$ be a topological space with distinguished point $e$. Then the abelian topological group $F(X)$ is said to be the Graev free abelian topological group on $X$ if

(i) $X$ is a subspace of $F(X)$ with $e$ the identity element of $F(X)$,

(ii) $X$ generates $F(X)$ algebraically, and

(iii) for every continuous map $\phi$ of $X$ into any topological group $G$ such that $\phi(e)$ is the identity of $G$, there exists a continuous homomorphism of $F(X)$ into $G$, which extends $\phi$.

The following theorem collects some known results about $F(X)$. (They are not the finest possible results but rather what we need here.) Recall first that a Hausdorff space $X=\bigcup_{n=1}^{\infty} X_{n}$ is said to be a $k_{\omega}$-space if each $X_{n}$ is compact and $X$ has the weak topology with respect to the $X_{n}$ 's.

THEOREM A. Let $X$ be any $k_{\omega}$-space. Then $F(X)$ exists [9], is connected [9], is a $k_{\omega}$-group [2], and hence is complete [2].

Received by the editors August 25, 1981.

1980 Mathematics Subject Classification. Primary 16A65, 22 A99.

Key words and phrases. Endomorphism ring, topological group, abelian group, representable ring.

(C) 1982 American Mathematical Society 
EXAMPLE. Let $I$ be the closed unit interval $[0,1]$. Let $F(I)$ be the Graev free abelian topological group on $I$ with distinguished point 0 . Then by the above theorem, $F(I)$ is a complete connected Hausdorff abelian topological group. Let $H$ be the subgroup of $F(I)$ generated algebraically by the rational numbers in $I$. Then $H$ is certainly countable, so $\operatorname{card}(H)<\operatorname{card}(F(I))$. Now define a countable family $\phi_{n}$ of continuous maps of $I$ into $F(I)$ by

$$
\phi_{n}(x)= \begin{cases}x, & x \leq 1 / n \\ 1 / n, & x>1 / n\end{cases}
$$

for each positive integer $n$. Then by the definition of free abelian topological group, each $\phi_{n}$ extends to a continuous endomorphism $\Phi_{n}$ of $F(I)$.

Now let $g \in F(I)$. Then $g=n_{1} g_{1}+n_{2} g_{2}+\cdots+n_{k} g_{k}$, for some $g_{i} \in I, g_{i} \neq 0$. Let $N$ be any positive integer such that $1 / N<g_{i}, i=1, \ldots, k$. Then

$$
\begin{aligned}
\Phi_{N}(g) & =n_{1} \phi_{N}\left(g_{1}\right)+n_{2} \phi_{N}\left(g_{2}\right)+\cdots+n_{k} \phi_{N}\left(g_{k}\right) \\
& =\left(n_{1}+n_{2}+\cdots+n_{k}\right) / n \in H .
\end{aligned}
$$

So no $g \in F(I)$ satisfies $\Phi_{n}(g) \notin H$, for all $n$.

So the answer to the stated problem is in the negative.

However Kabenjuk [3, 4, 5] and Wille [10] prove the following positive results.

THEOREM B. In a connected Hausdorff topological group of cardinality $2^{\aleph_{0}}$ every finite family of nontrivial continuous endomorphisms is small.

THEOREM C. Every countable family $\Phi$ of nontrivial continuous endomorphisms of a compact connected Hausdorff abelian group of countable weight is small.

We generalize Theorem $\mathrm{C}$.

THEOREM. Every countable family $\Phi$ of nontrivial continuous endomorphisms of a connected locally compact Hausdorff abelian group $G$ of countable weight is small.

ProOF. By Theorem 26 of [8], $G$ is topologically isomorphic to $\mathbf{R}^{n} \times K$, where $K$ is a compact connected topological group of countable weight, $\mathbf{R}$ is the additive topological group of real numbers, and $n$ is a nonnegative integer. Let $H$ be any subgroup of $G$ such that $\operatorname{card}(H)<\operatorname{card}(G)$. Then $H \leq H_{1} \times H_{2}$, where $H_{1}$ and $H_{2}$ are the projections of $H$ on $\mathbf{R}^{n}$ and $K$, respectively.

If $K$ is a nontrivial group, then being a connected compact Hausdorff group it has cardinality $\geq c$, the cardinality of the continuum. Then $\operatorname{card}(H)<\operatorname{card}(G)$ and $\operatorname{card}\left(\mathrm{H}_{2}\right) \leq \operatorname{card}(H)$ implies $\operatorname{card}\left(H_{2}\right)<\operatorname{card}(K)$. As $K$ is compact every continuous homomorphism of $K$ into $\mathbf{R}^{n}$ is trivial. Thus every member of $\Phi$ maps $K$ into itself. So by Theorem $\mathrm{C}$ above there exists an element $g \in K$ such that $\Phi g \cap H_{2}=\emptyset$. Thus $\Phi g \cap H=\emptyset$, as required.

If $K$ is the trivial group, then $G$ is $\mathbf{R}^{n}$. So every continuous endomorphism $\alpha \in \Phi$ is a linear transformation $\mathbf{R}^{n}$. As $H$ is countable, $\alpha^{-1}(H)$ is a countable union of cosets of the kernel, $K_{i}$, of $\alpha$. Let $\mu$ be Lebesgue measure on $\mathbf{R}^{n}$. As $\alpha$ is nonzero, $K_{\alpha}$ is at most an $(n-1)$-dimensional vector space and so $\mu\left(K_{\alpha}\right)=0$. Therefore $\mu\left(\alpha^{-1}(H)\right)=0$. Thus, since $\Phi$ is a countable family

$$
\mu\left(\bigcup_{\alpha \in \Phi} \alpha^{-1}(H)\right)=0 \text {. }
$$


So $\bigcup_{\alpha \in \Phi} \alpha^{-1}(H) \neq \mathbf{R}^{n}$. So choosing any $g \in \mathbf{R}^{n} \backslash \bigcup_{\alpha \in \Phi} \alpha^{-1}(H)$ we have that $\Phi g \cap H=\emptyset$, as required.

REMARK. Observe that in the second part of the above proof, dealing with $\mathbf{R}^{n}$, we assumed the continuum hypothesis.

In conclusion we note that our example showed that Kabenjuk's theorem cannot be extended from "compact" to "complete". However our example of the free abelian topological group on $[0,1]$ is certainly not a metrizable space and so we leave open the question of whether "compact" can be replaced by "complete metric".

\section{REFERENCES}

1. L. Fuchs, Infinite abelian groups. Vol. II, Academic Press, New York, 1973.

2. David C. Hunt and Sidney A. Morris, Free subgroups of topological groups, Proc. Second Internat. Conf. Theory of Groups, Canberra, Lecture Notes in Math., vol. 372, SpringerVerlag, Berlin and New York, 1974, pp. 377-387.

3. M. I. Kabenjuk, Finite groups of automorphisms of topological groups, Algebra and Logic 13 (1974), 291-299. (Russian)

4. __ Groups of automorphisms of topological groups, Algebra Symposium Proceedings. I, Gomel, 1975. (Russian)

5. _ Some topologies on abelian groups related to the ring of its endomorphisms, Colloquia Mathematica, Societatis János Bolyai 23 Topology (Budapest), 1978, pp. 705-712.

6. John Mack, Sidney A. Morris and Edward T. Ordman, Free topological groups and the projective dimension of a locally compact abelian group, Proc. Amer. Math. Soc. 40 (1973), 303-308.

7. Sidney A. Morris, Varieties of topological groups and left adjoint functions, J. Austral. Math. Soc. 16 (1973), 220-227.

8. __ Pontryagin duality and the structure of locally compact abelian groups, London Math. Soc. Lecture Notes Series, no. 29, Cambridge Univ. Press, London, 1977.

9. __ Varieties of topological groups, Bull. Austral. Math. Soc. 1 (1969), 145-160.

10. R. J. Wille, The existence of a topological group with automorphism group $C_{7}$, Quart J. Math. Oxford Ser. (2) 18 (1967), 53-57.

Department of Mathematics, Cleveland State University, Cleveland, OHIO 44115

DEPARTMENT OF MATHEMATICS, LA TROBE UNIVERSITY, BUNDOORA, Victoria 3083, Australia 